\title{
Embedding reward signals into perception and cognition
}

\author{
Luiz Pessoa ${ }^{\text {* }}$ and Jan B. Engelmann ${ }^{2}$ \\ 1 Department of Psychological and Brain Sciences, Indiana University, Bloomington, IN, USA \\ 2 Institute for Empirical Research in Economics, University of Zurich, Zurich, Switzerland
}

Edited by:

Anna C. Nobre, University of Oxford, UK

\section{Reviewed by:}

Tobias Egner, Duke University, USA

Anna C. Nobre, University of Oxford, UK

${ }^{*}$ Correspondence:

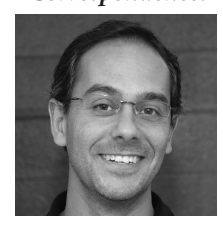

Luiz Pessoa received his B.Sc. and M.Sc. in Computer Science in Brazil, and a Ph.D. in Computational Neuroscience at Boston University (1996). From 1999 until 2003, he was a Visiting Fellow at the National Institute of Mental Health, Bethesda, in the laboratory headed by Leslie Ungerleider. After a few years at Brown University (2003-2006), he joined the Department of Psychological and Brain Sciences at Indiana University, Bloomington, where he is an Associate Professor. His interests center on the interactions between cognition and emotion/motivation in the human brain. lpessoa@indiana.edu

Despite considerable interest in the neural basis of valuation, the question of how valuation affects cognitive processing has received relatively less attention. Here, we review evidence from recent behavioral and neuroimaging studies supporting the notion that motivation can enhance perceptual and executive control processes to achieve more efficient goal-directed behavior. Specifically, in the context of cognitive tasks offering monetary gains, improved behavioral performance has been repeatedly observed in conjunction with elevated neural activations in task-relevant perceptual, cognitive and reward-related regions. We address the neural basis of motivation-cognition interactions by suggesting various modes of communication between relevant neural networks: (1) global hub regions may integrate information from multiple inputs providing a communicative link between specialized networks; (2) point-to-point interactions allow for more specific cross-network communication; and (3) diffuse neuromodulatory systems can relay motivational signals to cortex and enhance signal processing. Together, these modes of communication allow information regarding motivational significance to reach relevant brain regions and shape behavior.

Keywords: motivation, attention, executive function, fronto-parietal, posterior cingulate cortex

\section{INTRODUCTION}

Navigating through the world requires the constant assessment and reassessment of the value of our choices and actions. Making one choice may cause the loss of resources, such as food, but allow the attainment of others, such as mating. This harsh economic reality requires the brain to assess the costs and benefits of actions before committing to a motor response. Due to the ubiquity of such valuation processes and their importance for learning, action selection, and choice, there has been ample scientific interest in their neurobiological basis. This line of research has yielded results underscoring the importance of the dopamine system and its cortical projection sites in behavioral control (Schultz et al., 1992), has led to the formulation of computational models of valuation (Montague et al., 2004), and has contributed to the development of the novel field of neuroeconomics (e.g., Platt and Glimcher, 1999; Berns et al., 2001).

Despite much interest in the neurobiological basis of valuation processes, the question of how they interact with other cognitive systems has received relatively little attention. If valuation processes shape behavior, they should be expected to influence the perceptual and cognitive processes that are central to the production of behavior. Indeed, consistent with this notion, neuroimaging studies have shown that monetary incentives improve behavioral performance, concurrent with enhanced hemodynamic responses in task-relevant perceptual and cognitive regions, as well as regions of the reward system (e.g., Pochon et al., 2002; Small et al., 2005; Engelmann et al., 2009). Based on such findings, it has been proposed that the neural interaction between reward-related regions 
Reward system

Typically denotes a collection of interconnected structures that signal information related to rewards (among other functions). Major pathways include the mesolimbic and mesocortical dopamine systems, both of which originate in the ventral tegmental area and connect to multiple subcortical and cortical regions. Although we continue with the common practice of employing the term "reward system," it should be noted that its function is quite diverse (e.g., regulation of effort and resource allocation), subject to debate, and not restricted to reward per se.

Dprime ( $\left.d^{\prime}\right)$

Perceptual sensitivity measure commonly employed in signal detection theory. By taking into account both hits (e.g., correct target identification) and false alarms (e.g., incorrectly identifying distracters as targets) $d^{\prime}$ scores account for shifts in response criterion that are commonly observed under different reward conditions. with perceptual and cognitive networks that are task-relevant improves behavioral performance to maximize reward outcomes (Pessoa, 2009).

Executive processes, such as attention, working memory and inhibition, constitute a set of processes that are particularly important for behavioral planning and production. Given limited processing capacity, how can the brain separate those stimuli that deserve further processing from those that are better left ignored, to efficiently guide behavior? Traditionally, both bottom-up and top-down processes are posited as potential solutions to the "limited processing-resources dilemma" faced by the brain's executive system. By using top-down control, the brain can more efficiently allocate its resources based on current behavioral goals and prior knowledge. At the same time, processing resources should preferentially shift to salient features of the environment. Based on behavioral evidence, both of these processes are intimately linked to reward and motivation, as described below. Furthermore, these findings mesh well with previous demonstrations that the motivational dimensions of (top-down) goals rely on the dopamine system and its projection sites (e.g., Schultz et al., 1992). Interestingly (bottom-up) stimulus salience is also encoded in specific nodes of the reward system, such as the caudate nucleus (e.g., Zink et al., 2006).

\section{EFFECTS OF REWARD ON EXECUTIVE FUNCTION \\ PARAMETRIC INCREASES IN BEHAVIORAL PERFORMANCE AND ACTIVATION STRENGTH IN THE FRONTO-PARIETAL ATTENTIONAL NETWORK}

Here, we review evidence indicating that motivational factors guide perceptual and executive control processes, likely by modulating both bot- tom-up and top-down processes, thereby helping to solve the limited processing-resources dilemma. In a series of experiments, Engelmann and Pessoa (2007) and Engelmann et al. (2009) investigated the effects of motivation on task performance by probing the effects of parametric changes in incentive value on behavior during difficult spatial localization tasks. Participants were asked to indicate the location of a target stimulus (e.g., degraded face) relative to that of a distracter stimulus (e.g., random noise) as quickly and accurately as possible. Attention was manipulated by using a central cue that predicted target location with $70 \%$ validity (such that $30 \%$ of the time the cue indicated the incorrect target location) - in such cases, performance during validly cued trials is known to exceed that during invalidly cued ones. Motivation was parametrically manipulated in a blocked fashion by linking payoff to behavioral performance (if performance was both accurate and fast in a given block of trials, participants were given the chance to win cash incentives that varied from $\$ 0-\$ 4$, or to avoid losing money).

Our behavioral findings revealed improved detection performance as a function of absolute incentive value (Figure 1A). Critically, because behavior was characterized via the detection sensitivity measure $d^{\prime}$, the results revealed a "specific" effect of motivation on behavioral performance, instead of more unspecific influences such as arousal (e.g., purely faster response times) or response bias (e.g., more conservative responses) - but see below for further discussion on more general effects that may be, at least in part, linked to arousal. The same basic pattern of behavioral results was observed in three distinct versions of the task that varied in difficulty level,
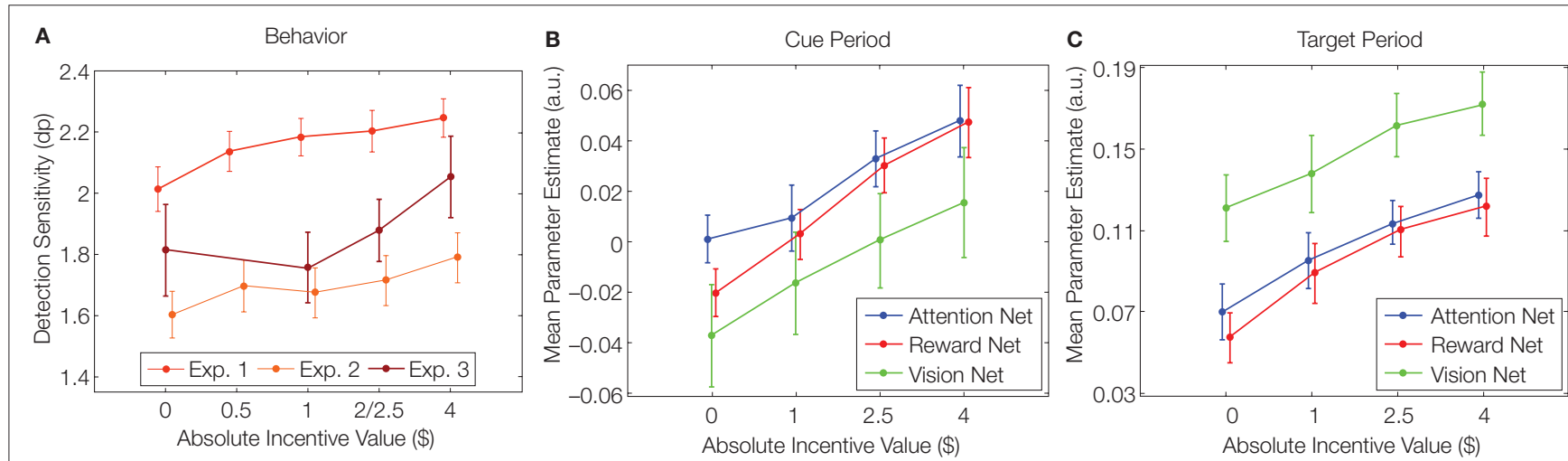

FIGURE 1 | Behavioral and neural effects of incentive motivation. (A) In all experiments, the detection sensitivity measure dprime (dp) increased as a function of absolute incentive magnitude. Red line: experiment 1 of Engelmann and Pessoa (2007); light orange line: experiment 2 of Engelmann and Pessoa (2007); dark red line: behavioral results of Engelmann et al. (2009).
Parallel increases in evoked brain responses observed in the study by Engelmann et al. (2009) during the cue (B) and target (C) task phases in three types of regions, namely attentional, visual and reward-related (see Figure 2 for some of the sites). Results were obtained by pooling the responses from regions within these three networks. Net = network. 


\section{Incentive motivation}

Present when an actor engages in effortful behavior to attain a valued goal. Incentives can be primary rewards, such as food items, or more abstract rewards, such as money. Often, highlyvalued incentives, such as career goals, require complex behaviors that demand sustained motivation over prolonged time periods. the type of target and distracter stimuli, and cue types (endogenous vs exogenous).

One of the versions of the behavioral task was accompanied by fMRI scanning (Engelmann et al., 2009), allowing us to probe the neural basis of enhanced performance by incentive motivation. Specifically, we sought to elucidate the workings of "process-specific" effects of motivation on cueand target-related processing during these attentional tasks. Non-specific motivational effects due to effort and arousal were removed by using a hybrid task design that included: (1) event-related (i.e., transient) components with relatively long, jittered and optimized intertrial and interstimulus intervals between cue and target periods; and (2) a blocked (i.e., sustained) motivational component. Hybrid designs allow for separate estimates of transient and sustained signals (Visscher et al., 2003). Importantly, transient processes could be dissociated from each other, i.e., cue- and targetrelated responses.

In parallel with the behavioral findings, the neuroimaging results revealed parametric increases in activation strength as a function of absolute incentive value in three types of brain regions (Figure 2): (i) fronto-parietal sites that are important for the control of attention, including

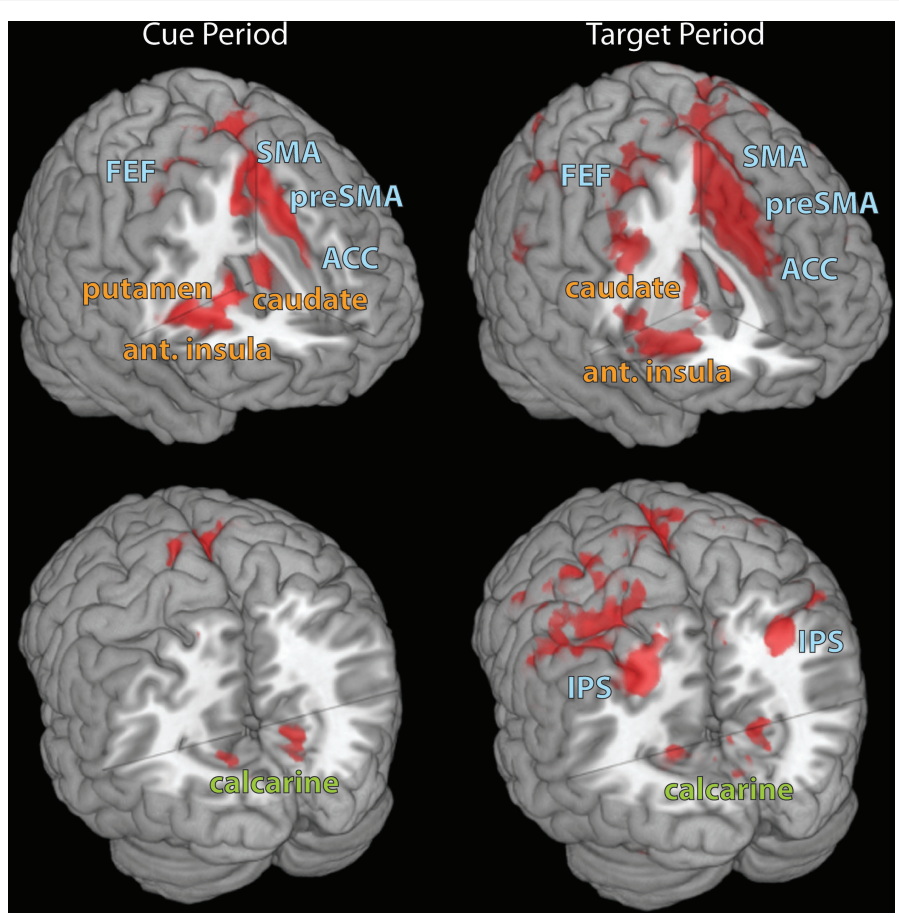

FIGURE 2 | Brain regions exhibiting correlations with absolute incentive magnitude during the cue and target task periods. Some of the attentional (blue font), visual (light green), and valuation (orange) regions are illustrated. ACC, anterior cingulate cortex; FEF, frontal eye field; IPS, intraparietal sulcus; pre-SMA, pre-supplementary motor area; and preSMA, pre-supplementary motor area. frontal eye field (FEF), anterior cingulate cortex (ACC; and other sites along the midline), intraparietal sulcus (IPS) and temporo-parietal junction (TPJ); (ii) occipito-temporal visual cortical sites, including sites around the calcarine fissure and in the fusiform gyrus, a region that is sensitive to face stimuli (which were employed in the task); and (iii) nodes of the reward system, including caudate and substantia nigra (SN)/midbrain. Parametric influences of incentive motivation on evoked responses were obtained during both the cue (Figure 1B) and target (Figure 1C) periods. In particular, our findings concerning rewardrelated sites are consistent with previous reports of parametric response increases in the nucleus accumbens (e.g., Knutson et al., 2005), caudate nucleus (Delgado et al., 2003) and orbitofrontal cortex (e.g., O’Doherty et al., 2001). Taken together, our observations revealed that parametric improvements in detection performance were accompanied by systematic modulations in three sets of brain regions that, together, support task performance, namely attentional, visual and reward-related regions.

\section{CONVERGING EVIDENCE: MOTIVATIONAL EFFECTS ON COGNITIVE AND SENSORY PROCESSING}

Our findings support the notion that motivational signals act both at more "central" levels in fronto-parietal cortex and at sensory levels. Here, we briefly discuss other studies that support this view and, in particular, have evaluated how motivation influences cognitive function. In a study by Small et al. (2005), fast target detection could lead to monetary wins or avoidance of monetary losses and, in the control condition, did not involve monetary outcomes. Better performance during the disengagement of attention was associated with enhanced activity in the inferior parietal lobe in the vicinity of the TPJ, a region that has been implicated in the reorienting of attention. Importantly, this effect was enhanced by incentive motivation during trials in which participants could win or avoid losing money, and were accompanied by activations in valuation-related regions, including the orbitofrontal cortex. Of particular interest, responses in the posterior cingulate cortex (PCC) were correlated with visual spatial expectancy (defined as the degree to which the cue benefited performance as evidenced by faster reaction times), an effect that was enhanced by incentive motivation. Given the known connectivity of this region with areas of the brain involved in attention and motivational processing, it was proposed that the PCC serves as a neural interface between motivation and the top-down control of attention. 
A subsequent study by Mohanty et al. (2008) also investigated motivation effects on attention, this time manipulating motivational state, namely hunger. Specifically, in the context of a covert-orienting task with a central cue, participants detected motivationally relevant (food) or irrelevant (tools) targets under conditions of hunger and satiety. As in the study by Small et al., responses in sites in parietal cortex (e.g., intraparietal sulcus, IPS) exhibited correlations with the speed of attentional shifts that were sensitive to not just motivational state, but also to the motivational value of the target. Similar patterns were also observed in the PCC and the orbitofrontal cortex (OFC). Furthermore, amygdala, PCC, locus coeruleus and $\mathrm{SN} /$ midbrain showed sensitivity to food-related cues when hungry, but not when satiated, an effect that did not generalize to tools. These findings demonstrate that motivational state (hunger) modulates spatial attention via response modulations across several brain regions.

Given that the findings from the above studies are being explicitly related to those of our own neuroimaging study, it is of relevance to ascertain the degree of spatial concordance of the parietal activation sites. In some cases, the concordance was good when compared to other attentional studies in the literature (Corbetta et al., 2000; Hopfinger et al., 2000; Kincade et al., 2005), such as target-related activations in the IPS (distance between our study and relevant published reports: $\sim 6 \mathrm{~mm}$ ). However, the concordance with the studies investigating attention and motivation per se (Small et al., 2005; Mohanty et al., 2008) was less impressive, such as $17 \mathrm{~mm}$ for the PCC, and even $\sim 23 \mathrm{~mm}$ for the TPJ. Hence, it will be important in the future to understand the reasons behind the sources of spatial variability.

The two studies reviewed above, in addition to our own work, provide evidence that motivation modulates fronto-parietal regions involved in attention. Additional evidence also supports the modulation of sensory cortex by motivation. For instance, Pantoja et al. (2007) investigated neuronal responses in the rat primary somatosensory cortex (S1) during a tactile discrimination task. Stimulus-related information encoded by $\mathrm{S} 1$ neuronal ensembles increased when the contingency between stimulus and response was crucial for reward, but not when reward was freely available. In addition, stimulus-related information was directly related to behavioral task performance. Related neuroimaging findings in humans were reported by Pleger et al. $(2008,2009)$, who used a tactile discrimination task coupled with financial rewards awarded for correct performance at the end of each trial. While reward improved discrimination performance and concordantly enhanced activity in the ventral striatum, the effect of reward on somatosensory responses was only observed in a post-stimulus phase between stimulus offset and reward delivery. Interestingly, the increase in somatosensory cortex responses varied parametrically as a function of reward magnitude. In addition, the effect of reward on somatosensory responses was mediated by the dopaminergic system, as evidenced via pharmacological manipulations (Pleger et al., 2009). As observed in our own study, the contribution of motivational signals to sensory processing extends to other sensory systems, with modulatory signals detected at the level of the primary visual cortex (V1) in both rats (Shuler and Bear, 2006) and humans (Serences, 2008). Thus, it appears that motivation not only modulates sensory processing, but that such influences are present at the first stages of cortical processing. Naturally, such effects likely reflect "late" contributions from other processing stages (see next section).

Thus far, we have reviewed motivational effects that appear to be more transient in nature; however, although relatively little is known about sustained motivational signals, such modulations have also been observed. For instance, in our experiment discussed above, we employed an experimental design in which incentive was manipulated in a blocked fashion, allowing us to investigate sustained responses throughout the block of trials and how they were modulated by motivation. State-like effects were observed in several brain regions, including sites in the prefrontal cortex (PFC; e.g., FEF, middle frontal gyrus), parietal cortex (e.g., IPS), in addition to the PCC. Related findings were also reported by Locke and Braver (2008) who reported increased sustained fMRI activity during rewarded blocks of a cognitive control task in a network of regions including the right lateral PFC, right parietal cortex, and dorsal medial frontal cortex. Importantly, in a recent study, Jimura et al. (in press) showed that the effect of an individual's sensitivity to reward on working memory performance was mediated by sustained effects of reward observed in the right lateral PFC. These studies highlight the importance of studying sustained effects of motivation, which may be more closely related to arousal processes. Indeed, future investigations seeking to unravel the contributions of both transient and sustained responses to behavioral performance are greatly needed.

\section{POTENTIAL MECHANIMS OF MOTIVATIONAL EFFECTS}

Our study, together with the ones cited above and several others, illustrates attempts at understanding how motivation influences cognitive and 
sensory processes. More generally, what are the neural bases for these effects? At the outset, it is instructive to consider the relationship between motivation and cognition more abstractly. For concreteness, we can consider attention as the cognitive task. We know that attention affects behavior, and one possibility is that motivation has similar effects that take place independently of attention (Figure 3A). A second scenario would suggest that motivation affects behavior by engaging the same set of processes that are used by attention. In this case, the impact of motivation on behavior could be described as mediated by attention (Figure 3B). This mediation could be partial only, such that both direct (motivation $\rightarrow$ behavior) and indirect (via attention) effects take place. Finally, it is possible to imagine situations in which attention and motivation are more highly interactive, such that they jointly influence behavior (Figure 3C). In this latter case, although one may choose to describe certain processes as "attentional" and others as "motivational", the interactions between the systems are sufficiently high, and their strict separation is possibly more semantic than real.

Methodologically, disentangling attention and motivation may be quite hard, and many effects attributed to motivation could be attentional and vice versa (Maunsell, 2004). Although separating their potential contributions is challenging, some evidence suggests that these processes may be partially dissociable. For instance, Bendiksby and Platt (2006) suggested that cell responses in the lateral intraparietal area (LIP) in the monkey exhibit separate contributions from reward and attention - in the context of a cued-saccade reaction time task that was coupled with blocked rewards. For example, neuronal activity was positively correlated with saccade reaction times, which in their task was considered to reflect the cost of attentional re-orienting, in a way that was independent of reward size. At the same time, modulation by reward size was independent of which type of cue appeared in the neuronal receptive field, with some cues being more predictive of target location than others, therefore putatively capturing more attention. Thus, their results are consistent with the idea that motivation and attention independently contribute to responses in LIP - although a stronger case for their separation would require converging evidence (e.g., in their study, other variables may have affected saccade reaction times, and not only attentional processes).

Both monkey electrophysiology and human neuroimaging research suggest that the control of selective attention relies on a distributed set of fronto-parietal regions, including FEF in frontal cortex and IPS in parietal cortex. These regions,
A Parallel

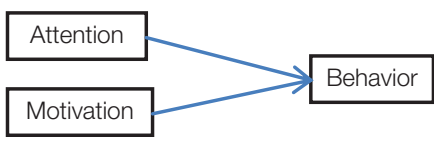

B Mediation

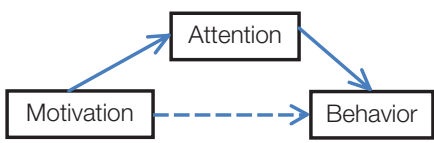

C Integration

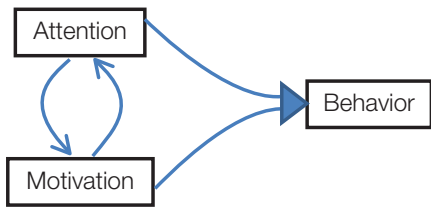

D Modes of communication

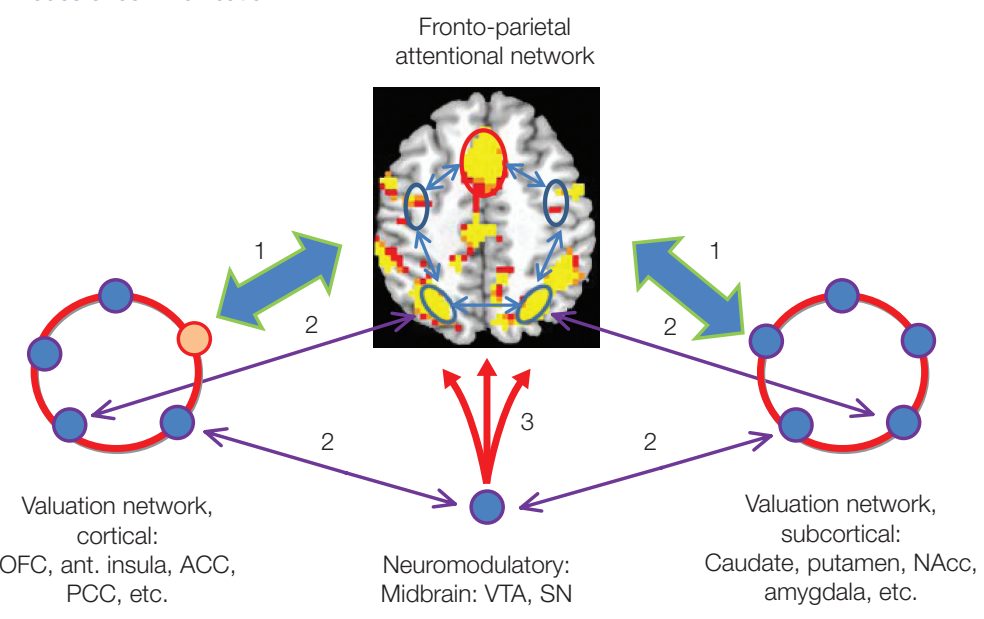

0

Connector hub region

Frontoparietal region
FIGURE 3 | Mechanisms of motivational effects on attention. (A-C) Potential, abstract relationships between attention and motivation and their effects on behavior. (D) Modes of communication between cognitive and motivation networks illustrated for attentional-motivational interactions. (1) Interactions rely on "hub" regions, such as the anterior cingulate cortex, which are part of both attentional and motivational networks (indicated via the red outline in both the valuation-cortical and attentional networks). (2) In addition, specific regions may link the two networks, either directly or via the thalamus. (3) Finally, motivational signals are embedded within cognitive mechanisms via the action of diffuse neuromodulatory systems. 
Hubs

Regions of high connectivity that have a disproportionately large impact on regulating information flow. Hubs are regions within neural networks that, through their privileged connectivity patterns, are able to integrate information and influence the processing of multiple connecting regions, thus greatly influencing brain dynamics. which in many cases appear to work together, are often conceptualized as "source" regions that exert control over sensory-processing areas to help select the information that is most relevant at a given time. One way to interpret the data from the attention studies described in the previous sections is to suggest that motivation acts on cognition to maximize potential reward in a way that relies on robust interactions between the attentional network and other reward/valuation networks. Among others, valuation regions include: (i) subcortically: the caudate, putamen and nucleus accumbens in the ventral striatum, and the amygdala; and (ii) cortically: the OFC, anterior insula, ACC and PCC. During trials in which reward (or punishment avoidance) is possible, valuation and attentional networks interact, resulting in enhanced behavioral performance that is supported by improved selection of sensory information. Critically, reward-related effects on cognitive function are specific (e.g., increased detection performance), as opposed to global (e.g., arousal). Whereas "independent" contributions from attention and motivation (Figure 3A) are not necessarily excluded, the above considerations are suggestive of the "mediation" and "integration" scenarios (Figures 3B,C) described above.

Indeed, we would like to propose that, more generally, the "integration" needs to be more seriously considered. Accordingly, the integration of motivational signals with those that are central to specific executive functions, including task switching, inhibition, and information maintenance, will rely on interactions between specific "cognitive" networks and those involved in determining the behavioral significance of the stimulus or task at hand. For instance, both dorsal (e.g., middle frontal gyrus) and ventral (e.g., inferior frontal gyrus) PFC sites, in addition to regions of parietal cortex (e.g., IPS), are important for maintaining and updating contextually relevant information "in mind." As in the case of the control of attention, we suggest that working memory-related signals are integrated with motivational ones in these areas. Consistent with this notion, cells in monkey lateral PFC not only hold information concerning an object's shape and location, but are also modulated by reward magnitude (Leon and Shadlen, 1999; Watanabe and Sakagami, 2007). Human neuroimaging studies have shown similar modulations of working memory signals in lateral PFC by reward (e.g., Pochon et al., 2002; Taylor et al., 2004). Furthermore, motivational information does not act simply as an "additive" mechanism; instead, in lateral PFC, cognitive and motivational signals appear to be integrated (see Jimura et al. (in press). For instance, in monkeys, during the delay period of a task involving spatial information, spatial and reward information do more than just add, as there is an increase of the amount of transmitted information concerning target position, as quantified by information theory (Kobayashi et al., 2002). In other words, reward information increases the discriminability of target positions, leading to enhanced performance. In the context of our own studies, in sharp contrast with other proposals (Kouneiher et al., 2009), we have suggested that the effect of motivation goes well beyond an "energizing" (i.e., a generalized "additive") function and, instead, involves enhancing and/or optimizing executive function (see also below) - a notion supported by the specific increases in detection sensitivity observed in our study; see also Small et al. (2005) and Mohanty et al. (2008).

The interaction between cognitive and motivation networks appears to take place via several modes of communication (Figure 3D). For instance, a specific brain region may function as a hub linking the two types of network. Recent advances in network theory (Guimera and Nunes Amaral, 2005) have shown that regions characterized by a high degree of connectivity, i.e., hubs (Sporns et al., 2007), are critical in regulating the flow and integration of information between regions. However, whereas the number of connections of a region is important in determining whether it will function as a hub, the structural topology of the region is also relevant. For instance, some regions are best characterized as "provincial" hubs (they occupy a central position within a single functional cluster; e.g., visual area V4, Sporns et al., 2007), whereas others act as "connector" hubs (they link separate region clusters). Hubs connecting cognitive and motivation networks would comprise examples of the latter type of region.

An intriguing suggestion by Mesulam et al. is that the PCC provides an important site for the integration of motivational and spatial attention information (Small et al., 2005; Mohanty et al., 2008; see also Platt and Huettel, 2008). In agreement with this suggestion in our neuroimaging study, as reviewed above, the PCC exhibited both motivation and attention signals. Specifically, not only did the PCC exhibit cue-related, targetrelated and sustained responses that increased with absolute incentive value, but increases in cue-related and sustained responses were correlated with individual trait measures tied to reward sensitivity (in this case, BAS-drive scores). Another, not mutually exclusive, possibility is that the ACC functions as a hub region linking the two types of network. The ACC is known to be important for integrating inputs from multiple 
sources, including affective and motivational inputs (Devinsky et al., 1995; Rushworth et al., 2007), and, in this respect, works in close cooperation with the anterior insula and OFC. The ACC has also been suggested to be involved in several executive processes, including conflict detection, error likelihood processing and error monitoring, and more generally helps determine the benefits and costs of acting. The ACC is also important for attentional control and controlling limitedprocessing capacity (Posner and DiGirolamo, 1998; Weissman et al., 2005; Pessoa, 2009). Thus, the ACC is a strong candidate for a hub connecting the two types of network.

In addition to interactions at specific connector hub regions, multiple "point-to-point" interactions may occur (indicated via the purple arrows in Figure 3D) that provide communication pathways between valuation and cognitive regions. For instance, in monkeys, the OFC projects to the ventral part of Brodmann area 46 on the lateral PFC surface (Barbas and Pandya, 1989). Another example includes the caudate nucleus, which is connected with several regions of frontal (including lateral PFC) and parietal cortices, in part via the thalamus (Alexander et al., 1986).

A third type of communication involves the diffuse action of neuromodulatory signals. Motivationally salient items engage dopaminergic cells in the ventral tegmental area (VTA) and SN. Widespread modulatory connections originating in these sites reach the entire cortical surface, thereby having the potential to rapidly influence cortical responses. Evidence from animal studies supports the notion that dopaminergic modulatory effects are associated with behavioral importance, generally (Schultz et al., 1992), and improve attentional accuracy, specifically (Granon et al., 2000; Seamans and Yang, 2004; Pezze et al., 2007). Several studies in humans, including ours, have also reported rewardrelated activation in dopaminergic centers (e.g., Bunzeck and Duzel, 2006; D’Ardenne et al., 2008) and, more commonly, their subcortical targets (e.g., caudate; both the head and body of the caudate have been reported). It is noteworthy that dopaminergic projections to the frontal lobe are much more significant than to posterior regions and, in particular, the occipital cortex appears to only minimally receive such projections (Oades and Halliday, 1987). These considerations are relevant to the understanding of the impact of motivation on both executive and sensory processes, and suggest that the impact of dopaminergic projection systems on visual function is likely to be relatively minor - though a complicating factor is that the effects could be strong though indirect. If this is correct, the effect on visual function reported in the studies above may be strongly dependent on "source" regions in frontal and parietal cortex that exert top-down modulatory signals on sensory processing.

Although neuromodulation can be viewed as simply another aspect of the suggested network interactions, it is worth separating it from the others for the following reasons. Because neuromodulatory signals target superficial (I-III) and deeper (V-VI) cortical layers, but tend to avoid layer IV (e.g., Raghanti et al., 2008), they appear to provide less of a "driving input" and instead may function to alter information processing. For instance, Goldman-Rakic et al. (1989) suggest that a major function of dopamine is to control cortical excitability, thereby possibly increasing the fidelity of signals computed within local networks (Douglas and Martin, 2007). More specifically, the effects of dopamine appear to enhance the neuronal signalto-noise ratio (Sawaguchi and Matsumura, 1985), consistent with computational modeling results of the role of dopamine in working memory function (Gruber et al., 2006). Thus, it is intriguing to suggest that dopaminergic neuromodulation may be a key mechanism by which motivation sharpens attention and behavioral performance, for instance via the enhancement of the signalto-noise ratio of relevant neurons. Therefore, the motivational context, which may be computed in valuation regions, may enhance the processing efficiency in cognitive regions via a dopaminergic signal. In the context of our task, valuation regions (e.g., OFC) signal behavioral relevance to neuromodulatory regions (e.g., VTA), which then enhance neuronal processing in relevant "cognitive" areas via dopamine signals (e.g., fronto-parietal regions). Future multi-site cell recordings may be able to more directly evaluate this working hypothesis. In any case, these specific effects on brain function are envisaged to be quite distinct from a simple "energizing" function.

Taken together, the available evidence suggests that motivation and cognition interact via multiple neural substrates to guide goal-directed behavior (Figure 3D). In particular, one or more of the above modes of communication may be operative at a given time depending on the particulars of the task at hand. More broadly, numerous opportunities for cognitive-emotional interactions exist in the brain, thereby allowing motivational significance to greatly shape complex behaviors.

\section{ACKNOWLEDGMENTS}

Support for this work was provided in part by the National Institute of Mental Health (R01 MH071589 to Luiz Pessoa). We also thank the reviewers for many valuable insights that have helped improve the paper. 


\section{REFERENCES}

Alexander, G. E., DeLong, M. R., and Strick, P. L. (1986). Parallel organization of functionally segregated circuits linking basal ganglia and cortex. Annu. Rev. Neurosci. 9, 357-381.

Barbas, H., and Pandya, D. N. (1989). Architecture and intrinsic connections of the prefrontal cortex in the rhesus monkey. J. Comp. Neurol. 286, 353-375.

Bendiksby, M. S., and Platt, M. L. (2006). Neural correlates of reward and attention in macaque area LIP. Neuropsychologia 44, 2411-2420.

Berns, G.S.,McClure,S.M.,Pagnoni, G., and Montague, P. R. (2001). Predictability modulates human brain response to reward. J. Neurosci. 21, 2793-2798.

Bunzeck, N., and Duzel, E. (2006). Absolute coding of stimulus novelty in the human substantia nigra/VTA. Neuron 51, 369-379.

Corbetta, M., Kincade, J. M., Ollinger, J. M., McAvoy, M. P., and Shulman, G. L. (2000). Voluntary orienting is dissociated from target detection in human posterior parietal cortex. Nat. Neurosci. 3, 292-297.

D'Ardenne, K., McClure, S. M., Nystrom, L. E., and Cohen, J. D. (2008). BOLD responses reflecting dopaminergic signals in the human ventral tegmental area. Science 319, 1264-1267.

Delgado, M. R., Locke, H. M., Stenger, V. A., and Fiez, J. A. (2003). Dorsal striatum responses to reward and punishment: effects of valence and magnitude manipulations. Cogn. Affect. Behav. Neurosci. 3, 27-38.

Devinsky, O., Morrell, M. J., and Vogt, B. A. (1995). Contributions of anterior cingulate cortex to behaviour. Brain 118(Pt 1), 279-306.

Douglas R. J., and Martin K. A. (2007). Recurrent neuronal circuits in the neocortex. Curr. Biol. 17, R496-R500.

Engelmann, J. B., Damaraju, E., Padmala, S., and Pessoa, L. (2009). Combined effects of attention and motivation on visual task performance: transient and sustained motivational effects. Front. Hum. Neurosci. 3, 4. doi:10.3389/neuro.09.004.2009.

Engelmann, J. B., and Pessoa, L. (2007). Motivation sharpens exogenous spatial attention. Emotion 7, 668-674.

Goldman-Rakic, P. S., Leranth, C., Williams, M., Mons, N., and Gefard, M. (1989). Dopamine synaptic complex with pyramidal neurons in primate cerebral cortex. Proc. Natl. Acad. Sci. U.S.A. 86, 9015-9019.

Granon, S., Passetti, F., Thomas, K. L., Dalley, J.W., Everitt, B. J., and Robbins, T.W. (2000). Enhanced and impaired attentional performance after infusion of D1 dopaminergic receptor agents into rat prefrontal cortex. J. Neurosci. 20, 1208-1215.
Gruber, A. J., Dayan, P., Gutkin, B. S., and Solla, S. A. (2006). Dopamine modulation in the basal ganglia locks the gate to working memory. J. Comput. Neurosci. 20, 153-166.

Guimera, R., and Nunes Amaral, L. A. (2005). Functional cartography of complex metabolic networks. Nature 433, 895-900.

Hopfinger, J. B., Buonocore, M. H., and Mangung, G. R. (2000). The neural mechanisms of top-down attentional control. Nat. Neurosci. 3, 248-291.

Jimura, K., Locke, H. S., and Braver, T. S. (in press). Prefrontal cortex mediation of cognitive enhancement in rewarding motivational contexts. Proc. Natl. Acad. Sci. U.S.A., early edition.

Kincade, J. M., Abrams, R. A., Astafiev, S. V., Shulman, G. L., and Corbetta, M. (2005). An event-related magnetic resonance imaging study of voluntary and stimulus-driven orienting of attention. J. Neurosci. 25, 4593-4604.

Knutson,B., Taylor,J., Kaufman,M.,Peterson, R., and Glover, G. (2005). Distributed neural representation of expected value. J. Neurosci. 25, 4806-4812.

Kobayashi, S., Lauwereyns, J., Koizumi, M., Sakagami, M., and Hikosaka, O. (2002). Influence of reward expectation on visuospatial processing in macaque lateral prefrontal cortex. $J$. Neurophysiol. 87, 1488-1498.

Kouneiher, F., Charron, S., and Koechlin, E. (2009). Motivation and cognitive control in the human prefrontal cortex. Nat. Neurosci. 12, 939-945.

Leon, M. I., and Shadlen, M. N. (1999) Effect of expected reward magnitude on the response of neurons in the dorsolateral prefrontal cortex of the macaque. Neuron 24, 415-425.

Locke, H. S., and Braver, T. S. (2008). Motivational influences on cognitive control: behavior, brain activation, and individual differences. $\operatorname{Cog} n$. Affect. Behav. Neurosci. 8, 99-112.

Maunsell,J.H.(2004). Neuronal representations of cognitive state: reward or attention? Trends Cogn. Sci. 8, 261-265.

Mohanty,A., Gitelman, D. R., Small, D. M., and Mesulam,M.M.(2008). The spatial attention network interacts with limbic and monoaminergic systems to modulate motivation-induced attention shifts. Cereb. Cortex 18, 2604-2613.

Montague, P. R., Hyman, S. E., and Cohen, J. D. (2004). Computational roles for dopamine in behavioural control. Nature 431, 760-767.

O’Doherty, J., Kringelbach, M. L., Rolls, E. T., Hornak, J., and Andrews, C. (2001). Abstract reward and punishment representations in the human orbitofrontal cortex. Nat. Neurosci. 4, 95-102.

Oades, R. D., and Halliday, G. M. (1987). Ventral tegmental (A10) system: neurobiology. 1. Anatomy and connectivity. Brain Res. 434, 117-165.
Pantoja, J., Ribeiro, S., Wiest, M., Soares, E., Gervasoni,D.,Lemos,N.A., and Nicolelis, M. A. (2007). Neuronal activity in the primary somatosensory thalamocortical loop is modulated by reward contingency during tactile discrimination. $J$. Neurosci. 27, 10608-10620.

Pessoa,L. (2009). How do emotion and motivation direct executive control? Trends Cogn. Sci. (Regul. Ed.) 13, 160-166.

Pezze, M.A., Dalley, J.W., and Robbins, T.W (2007). Differential roles of dopamine D1 and D2 receptors in the nucleus accumbens in attentional performance on thefive-choice serial reaction time task Neuropsychopharmacology 32, 273-283.

Platt, M. L., and Glimcher, P. W. (1999). Neural correlates of decision variables in parietal cortex. Nature 400, 233-238.

Platt, M. L., and Huettel, S. A. (2008) Risky business: the neuroeconomics of decision making under uncertainty. Nat. Neurosci. 11, 398-403.

Pleger, B., Blankenburg, F., Ruff, C. C., Driver, J., and Dolan, R. J. (2008). Reward facilitates tactile judgments and modulates hemodynamic responses in human primary somatosensory cortex J. Neurosci. 28, 8161-8168.

Pleger, B., Ruff, C. C., Blankenburg, F., Kloppel, S., Driver, J., and Dolan, R. J. (2009). Influence of dopaminergically mediated reward on somatosensory decision-making.PLoS Biol. 7,e1000164 doi: 10.1371/journal.pbio.1000164

Pochon, J. B., Levy, R., Fossati, P., Lehericy, S., Poline, J. B., Pillon, B., Le Bihan, D. and Dubois, B. (2002). The neural system that bridges reward and cognition in humans: an fMRI study. Proc. Natl. Acad. Sci. U.S.A. 99, 5669-5674.

Posner, M. I., and DiGirolamo, G. J. (1998). "Executive attention: conflict, target detection, and cognitive control," in The Attentive Brain, ed. R. Parasuraman (Cambridge, MA: MIT Press), 401-423.

Raghanti, M. A., Stimpson, C. D. Marcinkiewicz, J. L., Erwin, J. M. Hof, P. R., and Sherwood, C. C. (2008). Cortical dopaminergic innervation among humans, chimpanzees and macaque monkeys: a comparative study. Neuroscience 155, 203-220.

Rushworth, M. F., Buckley, M. J., Behrens, T. E., Walton, M. E., and Bannerman, D. M. (2007). Functional organization of the medial frontal cortex. Curr Opin. Neurobiol. 17, 220-227.

Sawaguchi, T., and Matsumura, M. (1985) Laminar distribution of neurons sensitive to acetylcholine, noradrenaline and dopamine in the dorsolateral prefrontal cortex of monkey. Neurosci. Res. 2, 255-273.

Schultz, W., Apicella, P., Scarnati, E., and Ljungberg, T. (1992). Neuronal activity in monkey ventral striatum related to the expectation of reward. J. Neurosci. $12,4595-4610$.
Seamans, J. K., and Yang, C. R. (2004). The principal features and mechanisms of dopamine modulation in the prefrontal cortex. Prog. Neurobiol. 74, 1-58.

Serences, J. T. (2008). Value-based modulations in human visual cortex. Neuron 60, 1169-1181.

Shuler, M. G., and Bear, M. F. (2006). Reward timing in the primary visual cortex. Science 311, 1606-1609.

Small, D. M., Gitelman, D., Simmons, K., Bloise, S. M., Parrish, T., and Mesulam, M. M. (2005). Monetary incentives enhance processing in brain regions mediating top-down control of attention. Cereb. Cortex 15, 1855-1865.

Sporns, O., Honey, C. J., and Kotter, R. (2007). Identification and classification of hubs in brain networks. PLoS ONE 2, e1049. doi:10.1371/journal. pone.0001049.

Taylor, S.F., Welsh, R. C., Wager, T.D., Phan, K. L., Fitzgerald, K. D., and Gehring, W. J. (2004). A functional neuroimaging study of motivation and executive function. Neuroimage 21, 1045-1054.

Visscher, K. M., Miezin, F. M., Kelly, J. E., Buckner, R. L., Donaldson, D. I., McAvoy, M. P., Bhalodia, V. M., and Petersen, S. E. (2003). Mixed blocked/ event-related designs separate transient and sustained activity in fMRI. Neuroimage 19, 1694-1708.

Watanabe, M., and Sakagami, M. (2007). Integration of cognitive and motivational context information in the primate prefrontal cortex. Cereb. Cortex 17(Suppl. 1), i101-i109.

Weissman, D. H., Gopalakrishnan, A., Hazlett, C. J., and Woldorff, M. G. (2005). Dorsal anterior cingulate cortex resolves conflict from distracting stimuli by boosting attention toward relevant events. Cereb. Cortex 15, 229-237.

Zink, C. F., Pagnoni, G., Chappelow, J. Martin-Skurski, M., and Berns, G. S. (2006). Human striatal activation reflects degree of stimulus saliency. Neuroimage 29, 977-983.

Conflict of Interest Statement: The authors declare that the research was conducted in the absence of any commercial or financial relationships that could be construed as a potential conflict of interest.

Received: 01 December 2009; paper pending published:24January 2010; accepted: 10March 2010; published online: 15 September 2010.

Citation: Pessoa L and Engelmann JB (2010) Embedding reward signals into perception and cognition. Front. Neurosci. 4:17. doi: 10.3389/fnins.2010.00017

Copyright $(2010$ Pessoaand Engelmann. This is an open-access article subject to an exclusive license agreement between the authors and the Frontiers Research Foundation, which permits unrestricted use, distribution, and reproduction in any medium, provided the original authors and source are credited. 